\title{
Helicobacter pylori dupA and smoking are associated with increased levels of IL-8 in
}

gastric mucosa in Iraq-reply

Thank you very much for your letter showing that full-length $d u p A$-type strains and smoking were independently associated with IL-8 levels. IL-8 mRNA levels are associated with polymorphonuclear neutrophil (PMN) infiltration and considered to be correlated with pathological change. Previously, we reported the importance of $d u p A$ in several papers $[1,2]$. Therefore their report was really interesting for us. We agree with the comments, and we plan to analyze the $\operatorname{dupA}$ genotype of strains isolated from Bhutan and the Dominican Republic. In our experiments, there was also no relationship between gender and mucosal IL-8 expression, in agreement with the Letter to the Editor by Hussein and Tuncel. As for smoking, the information from some patients was missing; however, we also try to gather the information. As pointed out, mean ages in our study were different between Bhutan and Dominican Republic. In our data, there was no significant relationship between age and mucosal IL-8 expression in both countries; therefore we evaluated IL-8 expression levels without adjusting ages between the two groups. It is true that many factors, including bacterial, environmental and host factors, are involved in IL-8 expression from gastric mucosa, and we should always take into account these factors as much as we can get the information.

\section{Acknowledgments}

This paper is based on the work supported in part by grants from the National Institutes of Health (DK62813), Grants-in-Aid for Scientific Research from the Ministry of Education, Culture, Sports, Science and Technology (MEXT) of Japan (22390085, 22659087, 24406015, 
24659200, 25293104 and 26640114), Strategic Young Researcher Overseas Visits Program for Accelerating Brain Circulation for Japan Society for the Promotion of Science (JSPS), the Strategic Funds for the Promotion of Science and Technology from Japan Science and Technology Agency (JST).

Hiroyuki Nagashima Yoshio Yamaoka MD, PhD Department of Environmental and Preventive Medicine Oita University Faculty of Medicine Yufu 879-5593, Japan and Department of Medicine-Gastroenterology Michael E. DeBakey Veterans Affairs Medical Center and Baylor College of Medicine Houston, Texas 77030, USA E-mail: yyamaoka@oita-u.ac.jp

\section{References}

[1] Lu H, Hsu P-I, Graham DY, Yamaoka Y. Duodenal ulcer promoting gene of Helicobacter pylori. Gastroenterology 2005;128:833-48.

[2] Jung SW, Sugimoto M, Shiota S, Graham DY, Yamaoka Y. The Intact $d u p A$ cluster is a more reliable Helicobacter pylori virulence marker than $d u p A$ alone. Infect Immun 2012;80:381-7. 